\title{
Estimation of Failure Probability of Smartphone's Battery Using Monte Carlo Simulation
}

\author{
Joon-Seong Lee Le a $^{1,2}$ \\ ${ }^{1}$ Dept. Of Mechanical System Engineering, Kyonggi University, \\ 154-42 Gwanggyosan-ro, Yeongtong-gu, Suwon, Gyeonggi-do, 443-760 Korea \\ ajslee1@kyonggi.ac.kr
}

\begin{abstract}
Keywords: Monte Carlo Simulation, Fuzzy Knowledge Processing, Failure Probability, Failure Assessment Diagram

Abstract. Battery is major component of smartphone, but only selected samples are periodically examined due to numerous numbers of batteries. The Consumers are generally required the use of high energy density batteries. However, most consumers are often dissatisfied with the battery life from even the most advanced lithium-ion rechargeable batteries in mobile phone. If cracks are not detected, such a cracking mechanism could lead to unstable rupture of the battery. Up to this time, integrity evaluations are performed using conventional deterministic approaches. So it is expected that the results obtained are too conservative to perform a rational evaluation of lifetime. In this respect, a probabilistic safety assessment method is more appropriate for the assessment of overall battery safety. This paper describes failure probability estimation of the battery using fuzzy knowledge processing. Failure assessment diagram (FAD) of battery material is proposed and applied in the probabilistic analysis.
\end{abstract}

\section{Introduction}

Loads Consumers are generally required the use of high energy density batteries. However, most consumers are often dissatisfied with the battery life from even the most advanced lithium-ion rechargeable batteries in mobile phone [1]. In recent years, plastic products have become thinner and lighter and proper materials, processing technology and product technology have been developed accordingly.

Since several incidents of leaking in the battery has been experienced, significant efforts have been made to improve the battery design, material, and fabrication upgrades during the last decade. Due to the uncertainty in inspection data, conservative data are used for the integrity evaluation. For example, lower bound in fracture toughness and upper bound in stress data, crack growth rate are used. It results in, therefore, difficulty in estimating lifetime. The probabilistic approach would be a suitable way for the evaluation in taking into account the uncertainties associated with important integrity parameters, such as inspection sampling sizes, flaws distribution. Many outstanding probabilistic research works have been conducted especially for battery in the event of thermal shock. However, since the probabilistic integrity assessment of batteries requires more complicated input data, only limited studies were so far performed.

For realistic analysis failure criteria, which is more suitable for probabilistic analysis [2], is proposed based on failure assessment diagram (FAD) [3]. In this paper, the evaluation of failure probabilities in battery considering thermal mechanism was investigated using fuzzy knowledge processing. To determine the failure of battery, FAD is applied.

\section{Principal Algorithms}

Designation of Node Density Distribution. In the present system, nodes are first generated, and then an FE mesh is built. In general, it is not so easy to well control element size for a complex geometry. A node density distribution over a whole geometry model is constructed as follows. The present system stores several local node patterns such as the pattern suitable to well capture stress concentration, the pattern to subdivided a finite domain uniformly, and the pattern to subdivide a 
whole domain uniformly. An user selects some of those local node patterns, depending on their analysis purposes, and designates their relative importance and where to locate them. When designers do not want any special meshing, they can adopt uniformly subdivided mesh.

Failure Criteria. Battery material has enough ductility at installation because it was made by cold-work. During operation it loses ductility due to hydride precipitation and irradiation embrittlement. Current failure criteria are unstable fracture and plastic collapse for the consideration of material behavior change. However, some test data cannot be explained by these failure criteria.

More exact failure criteria are required because no safety margin is applied in probabilistic analysis. FAD can explain these data more exactly. For the application of FAD [3], failure parameters such as stress intensity factor $\left(\mathrm{K}_{\mathrm{I}}\right)$, fracture toughness $\left(\mathrm{K}_{\mathrm{IC}}\right)$, plastic collapse stress $\left(\sigma_{\mathrm{c}}\right)$ and applied stress $\left(\sigma_{\mathrm{a}}\right)$ are used. In this study the FAD is used for the consideration of material behavior change.

As shown in Fig. 2 that is FAD, straight line (box) is current failure criteria, whereas FAL is failure criteria used in this study. Current criteria cannot explain region " $B$ " in Figure 3 . In the FAD, two data sets are displayed: one is derived from actual tensile strength and fracture toughness, and the other from mean value of tensile strength and fracture toughness. Failure assessment line (FAL) used in FAD is shown in Eqs. (1) (3).

$$
\begin{gathered}
K_{r, F A L}=\left[1+\frac{L_{r}^{2}}{2}\right]^{-1 / 2} \times\left[0.3+0.7 \exp \left(-0.6 L_{r}^{6}\right)\right] \\
K_{r}=K_{I} / K_{I C} \\
L_{r}=\sigma_{a} / \sigma_{c}
\end{gathered}
$$

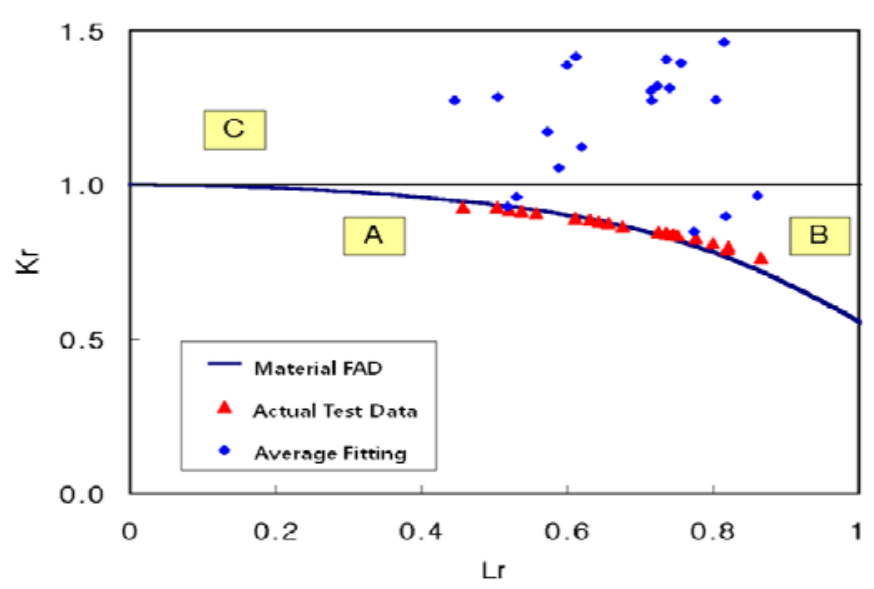

Fig. 2 Failure assessment line versus bust test data

\section{Probabilistic Integrity Assessment}

Among various probabilistic approaches, Monte Carlo (MC) method [5] is used in this study to evaluate failure probabilities as a function of operation time. Some probabilistic variables are selected according to an employed analysis model. Probabilistic variables include initial crack size, material properties and so on. Crack growth simulations are performed by deterministic fracture mechanics considering inspection and transient data. Finally, failure probabilities are calculated as a function of operation time. In MC simulation the convergence was obtained when number of iterations equal to $10^{6}$. In order to decrease the random number effect, five independent simulations are carried and an average value is used as a result. 
To check the failure criteria effect, some case studies were carried out. Probabilistic density function (PDF) [6] for each probabilistic variable assumed in this study was derived from inspection data and testing data. Details of probabilistic are shown in Table 1.

Table 1 Details of probabilistic variables

\begin{tabular}{|c|c|c|c|c|c|}
\hline Probabilistic variables & PDF type & Mean & S.T.D & Min. value & Max. value \\
\hline Aspect ratio (a/c) & Exponential & 0.12 & N.A. & 0.1 & 1.0 \\
\hline Depth ratio (a/t) & Log-normal & 0.10 & 0.08 & 0.01 & 0.5 \\
\hline Fracture toughness & Log-normal & 67.0 & 12.0 & 20.0 & 120.0 \\
\hline
\end{tabular}

\section{Examples and Discussions}

Fig. 3 shows the example of the application of this mesh generator for three-dimensional geometry. As shown in figure, a uniform mesh and a nonuniform mesh were connected very smoothly. The mesh consists of 10,108 tetrahedral elements and 12,442 nodes. Nodes and elements are generated in about 5 minutes and in about 2 minutes, respectively. Fig. 4 shows the protection module package (PMP) [7] of smartphone's battery.

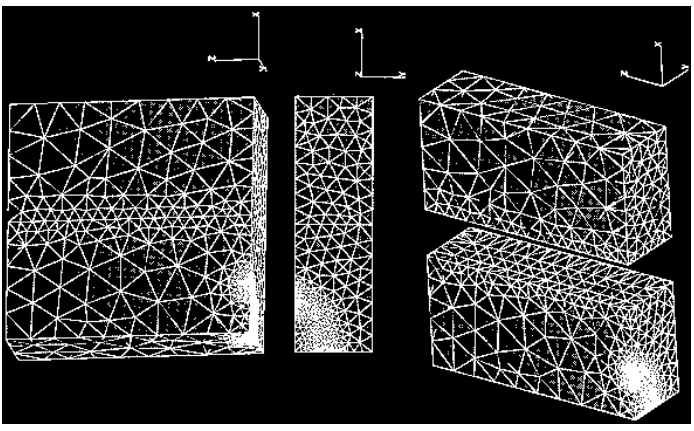

Fig. 3 A typical mesh

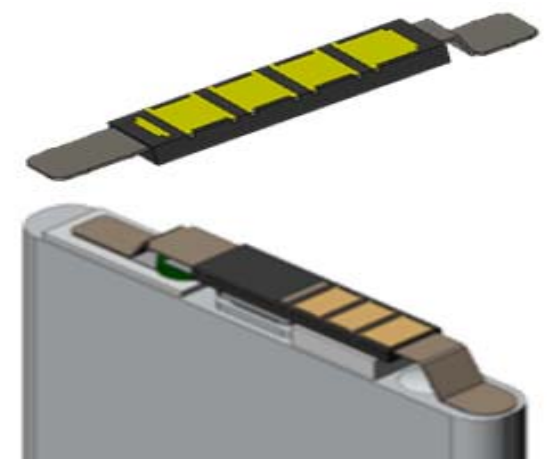

Fig. 4 Protection module package of battery

To verify the proposed FAD in this study, four analyses are performed. The Zahoo's equation is used to calculate the global plastic collapse stress whereas Carter's equation is used to local collapse. Leak and failure probabilities are shown in Figures 5 and 6. For all cases failure or leak probabilities are less than $10^{-5}$ during design life. But five-year difference was observed as wall penetration criteria change as shown in Fig. 5. When crack depth reaches to $80 \%$ of thickness, the leak probability between the local collapse and the global collapse, which is used by failure criteria, has no difference almost. In case crack depth reaches to $100 \%$ of thickness, however, analysis result of two failure criteria shows some measure difference. The leak probability is reached in allowable failure probability after 3.8 and 4.3 years, respectively.

\section{Summary}

A probabilistic fracture mechanics methodology which could evaluate the integrity of smart phone's battery using fuzzy knowledge processing and MC simulation was developed. Analyses about failure probability are performed using probabilistic variables taken from inspection data. It is known that the governing failure mode was leakage or plastic collapse in all cases analyzed in this study, whereas unstable fracture was governing failure mode in deterministic analysis. Also, sensitivity analyses were conducted for evaluating battery. For more realistic failure estimation FAD are applied instead of current failure criteria that explains test data more exactly. 


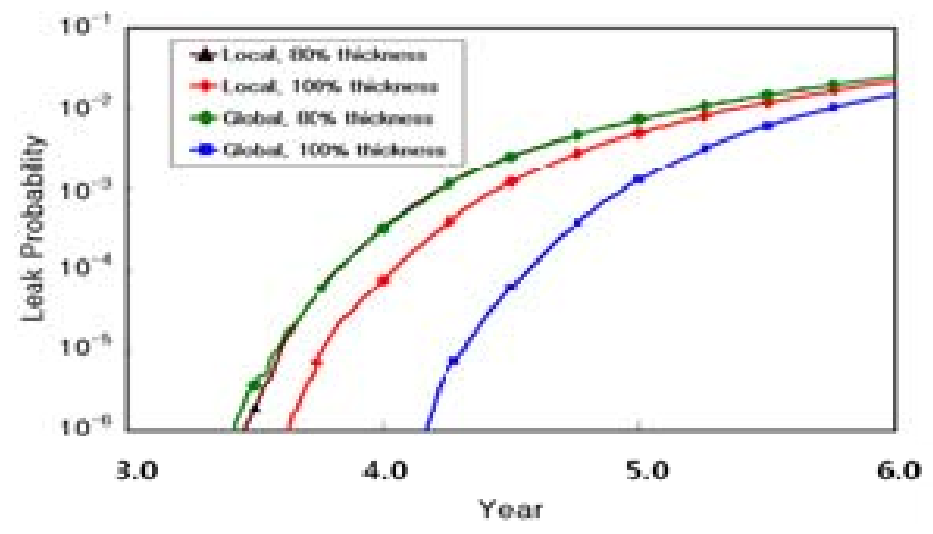

Fig. 5 Leak probability for various condition

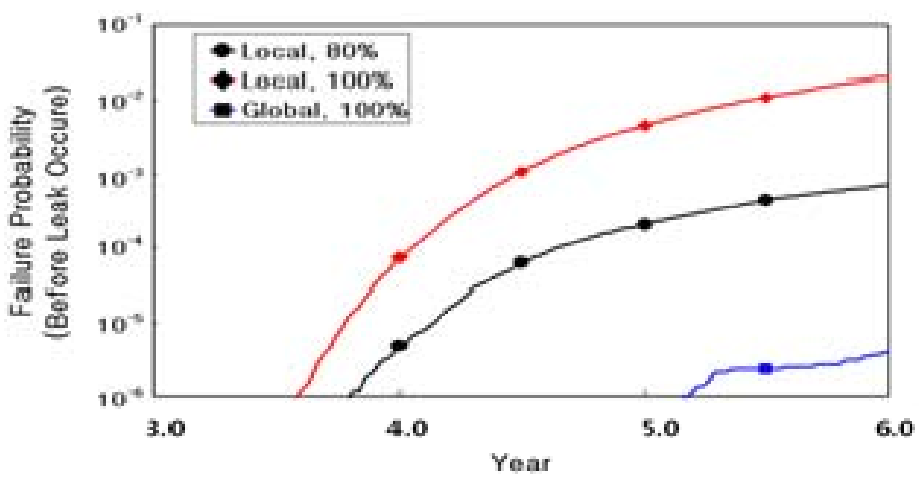

Fig. 6 Failure probability for various condition

\section{References}

[1] Watson, J.S. Lee, H.R. Hong, G.H. Jo and D.K. Park,"Strength Analysis of Epoxy Molding Compound Module Using Automated FEA System", Applied Mechanics and Materials, Vol. 670-671, 2014.

[2] J.S. Lee, S.L. Kwak, J.S. Lee, Y.J. Kim, "Probabilistic Integrity Assessment of Axial Flaw in CANDU Pressure Tube Due to the Diameter and Thickness Variation", Fitness for Service Evaluations and Non-Linear Analysis, Proceedings of the 2002 ASME Pressure Vessels and Piping Conference, Vancouver, Canada, PVP-Vol. 442, pp. 111-118, 2002.

[3] J.S. S.L. Kwak, J.S. Lee, Y.J. Kim and Y.W. Park, "A Probabilistic Integrity Assessment of Flaw in Zirconium Alloy Pressure Tube Considering Delayed Hydride Cracking”, Advanced Materials Development \& Performance (AMDP), Proceedings of the International Conference AMDP 2002, CD-rom, 2002.

[4] J.S. Lee, "Automated CAE System for Three-Dimensional Complex Geometry", Doctoral Thesis, The University of Tokyo, 1995.

[5] J.S. Lee, S.L. Kwak and Y.J. Kim, "Application of Probabilistic Fracture Mechanics Technique Using Monte Carlo Simulation”, Journal of the Korean Society for Precision Engineering, Vol. 18, No. 10, pp. 154-160, 2001.

[6] J.S. Lee and H.J. Lee, "An Automated CAE System for Multidisciplinary Structural Design: Its Application to Micro Accelerometer", J. of Mechanical Science and Technology, 24(9), pp. 1875-1883, 2010.

[7] J.S. Lee, H.R. Hong, G.H. Jo and D.K. Park, "Strength Analysis of Epoxy Molding Compound Module Using Automated FEA System", Applied Mechanics and Materials, Vol. 670-671, pp. 852-855, 2014. 